\title{
Significant proportion of acute hepatitis B in Poland in 2010-2014 attributed to hospital transmission: combining surveillance and public registries data
}

\author{
Małgorzata Stępień* (D), Karolina Zakrzewska and Magdalena Rosińska
}

\begin{abstract}
Background: Efficient control of acute hepatitis B requires identification of current transmission routes. Countries in Central-Eastern Europe including Poland attribute an important fraction of cases to nosocomial transmission, as opposed to Western European countries. However, due to possible multiple exposures during the incubation time such assignment may be debatable. This study aimed at assessing of most affected groups and current transmission pattern of acute hepatitis B.
\end{abstract}

Methods: We investigated exposures reported by acute hepatitis B cases notified to routine surveillance system in Poland in 2010-2014 in comparison to data on hospitalization rates in general population.

Results: Hospitalization during incubation time significantly increased the risk of HBV infection (RR 3.13,95\% Cl 2.58-3.80). Overall hospitalization population attributable risk (PAR\%) was 25.7\% (95\% Cl 20.3\%-31.1\%) as compared to 35\% of acute cases assigned to hospital transmission in surveillance database.

PAR\% increased from 9.5\% (1.12\%-17.8\%) in the age group $25-34$ to $41.1 \%(28.2 \%-53.9 \%)$ among those $65+$. In addition, cases $<40$ more frequently than the older ones reported history of injecting drugs and risky sexual contacts ( $25 \%$ vs $5 \%$ ). $27 \%$ of men $<40$ did not report any exposure at all, drawing attention to possible underreporting of risk behaviors.

Conclusions: The distribution of probable transmission routes differed by age and gender. Further improvement of HBV control requires better coverage of vaccination in risk groups but also strengthening the blood-borne infections control in hospitals.

Keywords: Hepatitis B, Epidemiology, Incidence, Surveillance, Vaccination, Modes of transmission

\section{Background}

Hepatitis B continues to be a serious public health problem worldwide. According to the WHO estimates approximately 257 million people are infected with hepatitis B and over 880 thousands died in 2015 because of the late consequences of this infection [1].

Introduction of an effective vaccine against hepatitis B in the 1980s, significantly reduced incidence of this disease in many countries over the world. In Europe, depending on the epidemic situation, routine immunization of

\footnotetext{
* Correspondence: mstepien@pzh.gov.pl

Department of Epidemiology, National Institute of Public Health - National

Institute of Hygiene, Chocimska 24, 00-791 Warsaw, Poland
}

infants and/or targeted vaccination of risk groups was adopted [2].

Reported transmission routes differ between countries across EU. While over $40 \%$ overall, are attributed to sexual transmission [3], in some countries, especially in Central-Eastern Europe, considerable proportion of cases is thought to be due to nosocomial transmission. Whether or not it is really the case is debatable, due to long incubation period and possibly multiple exposures during this time. There is little information about possible molecular and/or environmental investigation of patient-to-patient transmission events from this region. In a review of published patient-to-patients transmission 
events in the EU and the USA, none of the analyzed events was reported from this region [4].

Poland was one of the countries with the highest hepatitis $B$ incidence in Europe. The first noticeable decline was observed in 1986 attributed to the improvement of the medical equipment sterilization procedures, particularly gradual replacement of dry air sterilizers with autoclaves [5]. However, in early 1990s there were still 35 cases observed per 100,000 population, as compared to the European average of approximately19/100,000 at that time [5-7]. A real breakthrough occurred after the introduction of universal vaccination of newborns against hepatitis B in 1994-1996 [8, 9]. Later on, in 2000, a catch-up vaccination for adolescents aged 14 was introduced. In effect, the coverage among the birth cohorts born in 1986 or later as well in other groups targeted by immunization program, particularly health care workers exceeds 99\% [9]. In adults born before 1986, vaccinated mostly in relation to planned surgeries or chronic diseases, the coverage is estimated at about 15$17 \%[8,10,11]$, although recent studies among attendees of primary health care suggest even higher values, exceeding 50\% [10]. Nonetheless, acute hepatitis B continues to occur in the unvaccinated cohorts. The number of new acute infections depends also on the current prevalence of $\mathrm{HBV}$ infections which remain a source of new infections for the unvaccinated population. Prevalence of HBsAg reported in recent studies range from $0.9 \%$ to $1.12 \%$. However, these data originate from studies conducted on subpopulations or small groups of people $[12,13]$. An important source of information are data on HBV infections among blood donors - in the monitoring of blood donation within 20 years there was a decrease in the prevalence of HBsAg both among first-time (from $1 \%$ in 1994 to $0.3 \%$ in 2013) and repeat donors $(0.1 \%$ to $0.02 \%)$ [14].

In addition to blood donors, all pregnant women, patients undergoing chronic dialysis, HIV-infected individuals, and persons applying for the refugee status are routinely screened for HBsAg. Existing care pathways allow to link all diagnosed individuals into appropriate care, including treatment with NA and PegIFNa2a.

Sustainable improvement of the epidemiological situation of hepatitis B depends on better understanding of the transmission patterns, both to target adult vaccination and to implement or improve other control measures. In surveillance data $35 \%$ of acute hepatitis B are attributed to hospital transmission and further $23 \%$ to medical procedures in the outpatient setting, although the evidence for such assignment is generally lacking (NIZP-PZH, unpublished data).

The aim of this study is to identify demographic groups in Poland who are the most affected with acute hepatitis B. Importantly, we compare the incidence among people, who were hospitalized during the past year and those who were not in order to quantify the role of potential transmission in the hospital settings. We use routinely collected data including infectious disease surveillance data as well as other public registries data, allowing to replicate the study over the time.

\section{Methods}

\section{Data sources}

The evaluation of the epidemiological situation was based on data on acute hepatitis B cases collected through the routine mandatory surveillance system in Poland between 2010 and 2014 (individual case reports). This period was selected to assure comparability of data, with respect to the case definition and the data sources. Over the analyzed period the system was comprehensive and based on reporting by clinicians. Reporting form for clinicians requires determining on what basis the disease was diagnosed (antiHBc IgM, symptoms, epidemiological link or other). Cases reported as acute who did not meet the criteria required for confirmed or probable acute hepatitis $B$ were qualified as unknown HBV (as to phase) and were not included in this study. Since April 2014, reporting of the positive test results by laboratories was introduced as parallel data source. In addition to the clinical report supplementary epidemiological data were collected (among other the history of exposures) by the local epidemiologists on a standardized form. Data on hospitalization derived from annual reports of National Healthcare Found publicly available from: http://www.nfz.gov.pl/zarzadzenia-prezesa/uchwaly-radynfz/uchwala-nr-42015ii,6343.html.

\section{Acute hepatitis B case definition}

Cases of hepatitis B were reported to the State Sanitary Inspection by physicians who diagnose or suspect the disease on the basis of existing legislation. In 20102013, cases meeting the criteria of the case definition for acute hepatitis B approved by the EC in its decision of 2008/426/EC [15] for reporting communicable diseases to the community network were registered. According to this definition, acute cases were all symptomatic cases, laboratory confirmed by the presence of anti-HBc IgM and probable acute cases were symptomatic cases epidemiologically linked to a confirmed case of hepatitis B. In 2014 a new case definition for hepatitis B (2012/ $506 / \mathrm{EU})$ was implemented with minor modifications to routine surveillance in Poland. In comparison to previous case definition, this introduced in 2014 excluded probable acute cases and laboratory criteria (positive results for specific anti-HBc IgM antibodies) were the only basis for the registration of acute hepatitis $\mathrm{B}$ case. 


\section{Probable transmission route}

The following exposures during 6 months prior to onset were ascertained through the reporting form: a) medical invasive and minimally invasive procedures by setting (hospital, outpatients clinic or nursing home), b) nonmedical including: drug use (especially injecting use), home contact with HBV infected person, sexual contact with HBV infected person, sexual contact with people who inject drugs (PWID) or men who have sex with men (MSM) or multiple partners, tattooing, piercing, acupuncture, beauty treatments with skin puncturing, birth by a mother HBV infected during pregnancy, c) occupational exposure in medical or non-medical professions. Based on reported exposures probable transmission route was specified: medical procedures, PWID, sexual transmission including contact with HBVinfected and high risk sexual contacts, household contact with HBV-infected person, tattooing / piercing / beauty treatments, others (including occupational infections) or unknown/no data. In cases who reported more than one exposure the route of infection was classified into the above categories on the basis of the following hierarchy of risk factors: PWID, MSM, occupational exposures, chronic dialysis, heterosexual contact with a person infected with HBV, home contact with a person infected with $\mathrm{HBV},>1$ sexual partner (heterosexual) during past 6 months and other including tattoo, piercing and beauty treatments. Since the risks associated with performing individual medical procedures are not well defined, in the case of multiple exposure medical procedures or hospital stay were set at the end of the list. The applied hierarchy was based on hierarchy proposed by Goldstein S [16] with modifications: we excluded blood transfusion from an infected donor, because after introduction of donor routine tests for HBV-DNA in 2005 risk associated with transfusions was reduced to a minimum $[14,17]$.

\section{Hospitalization}

Hospitalization was defined as hospitalization due to any reason, but excluding nursing homes. For cases, the annual number of hospitalized in a given year was calculated as number of cases with the disease onset within this year reporting hospitalization during the presumed incubation period, i.e. within the 6 months prior to onset. For the general population we used a registered number of people who were hospitalized during a calendar year.

\section{Statistical analysis}

Statistical analysis was restricted to cases in the unvaccinated birth cohorts (born before 1986). Average annual incidence was calculated using the reported cases and the available denominator estimates from the country statistics. Incidences by sex, hospitalization and employment status were compared using Student T-test or Chisquare test.

We calculated risk ratios (RRs) and 95\% confidence intervals (CIs) for hospitalization, stratifying by the year of onset and age group. The population attributable risk (PAR\%) and 95\% confidence intervals for it was also calculated [18]. Chi-square test was applied for comparing category distribution, significance was defined as $p$ value $\leq 0.05$. Statistical analysis was performed using EpiInfo 3 . 5.3 .

\section{Results}

In total, 459 cases of acute hepatitis B were recorded within 5 years, including 19 cases from vaccinated birth cohorts who were discussed separately. The incidence in the analyzed period decreased from 0.34 per 100,000 in 2010 to 0.2 in 2014. Among all cases 65\% were men, and 39\% resided in larger cities (> 100,000 inhabitants). The incidence distribution by age was markedly different in men and women. It was increasing with age in women. In contrast, among men there was a distinct peak in the age group 25-34 (Fig. 1). Additionally, the incidence was higher among the unemployed than among working population (0.46 vs 0.25 per 100,000) and among those hospitalized in the past 12 months than among those who were not (0.71 vs 0.22$)$. Table 1 presents the indicators in birth cohorts unvaccinated in infancy.

The incidence rate among hospitalized was significantly higher for all the analyzed years, relative risk (RR) reaching the highest values in $2014(\mathrm{RR}=4.20,95 \%$ CI 2 . 56-6.88) (Table 2). The RR was significant for all age groups, lower for the age group 25-34, 1.86 (95\% CI 1. 19-2.91) as compared to the older age groups, among whom it ranged from 3.42 to 3.90 . Overall population attributable risk (PAR\%) was 25.7\% (95\% CI 20.3\%-31.1\%) and it increased from $9.5 \%(1.12 \%-17.8 \%)$ in the age

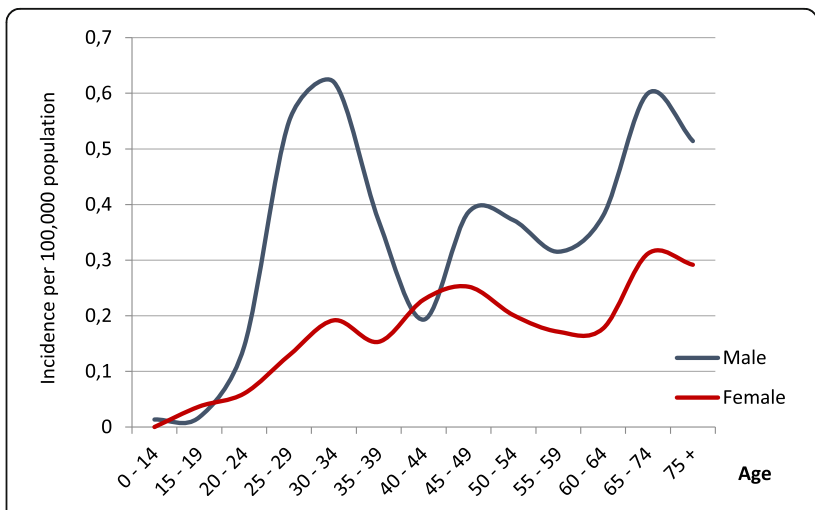

Fig. 1 Acute hepatitis B in Poland in 2010-2014. Incidence per 100,000 population by age and gender 
Table 1 Acute hepatitis B in Poland in 2010-2014 in birth cohorts unvaccinated in infancy. Distribution of reported cases and incidence by demographic features and history of recent hospitalization

\begin{tabular}{|c|c|c|c|c|c|}
\hline & & $\mathrm{N}$ & $\%$ & Incidence per 100,000 & $p$-value $e^{a}$ \\
\hline Total & & 440 & 100 & 0.33 & \\
\hline \multirow[t]{5}{*}{ Onset year } & 2010 & 123 & $28.0 \%$ & 0.45 & 0.822 \\
\hline & 2011 & 101 & $23.0 \%$ & 0.37 & \\
\hline & 2012 & 74 & $16.8 \%$ & 0.28 & \\
\hline & 2013 & 79 & $18.0 \%$ & 0.30 & \\
\hline & 2014 & 63 & $14.3 \%$ & 0.24 & \\
\hline \multirow[t]{2}{*}{ Sex } & Male & 285 & $64.8 \%$ & 0.45 & 0.0011 \\
\hline & Female & 155 & $35.2 \%$ & 0.22 & \\
\hline \multirow[t]{4}{*}{ Birth cohort-group ${ }^{b}$} & 1976-1985 & 133 & $30.2 \%$ & 0.42 & $<0.001$ \\
\hline & $1961-1975$ & 96 & $21.8 \%$ & 0.25 & \\
\hline & $1946-1960$ & 113 & $25.7 \%$ & 0.28 & \\
\hline & born before 1946 & 98 & $22.3 \%$ & 0.42 & \\
\hline \multirow[t]{4}{*}{ Place of residence } & Urban total & 304 & $69.1 \%$ & 0.35 & $0.0156^{c}$ \\
\hline & Cities $<100,000$ & 129 & $29.3 \%$ & 0.28 & $0.0149^{d}$ \\
\hline & Cities $\geq 100,000$ & 175 & $39.8 \%$ & 0.42 & \\
\hline & Rural & 136 & $30.9 \%$ & 0.26 & \\
\hline \multirow[t]{4}{*}{ Professional activity (adults) } & Employed & 191 & $43.4 \%$ & 0.26 & $<0.001$ \\
\hline & Unemployed & 37 & $8.4 \%$ & 0.58 & \\
\hline & Retirees & 117 & $26.6 \%$ & 0.37 & \\
\hline & Disability pensioners & 42 & $9.5 \%$ & 0.55 & \\
\hline Hospitalization history & Not hospitalized & 274 & $62.3 \%$ & 0.24 & $<0.001$ \\
\hline (during the incubation period) & Hospitalized & 166 & $37.7 \%$ & 0.74 & \\
\hline
\end{tabular}

${ }^{a} X^{2}$ or Hartley's $f$ test

${ }^{\mathrm{b}}$ Born after 1985 were vaccinated in infancy or at age 14

'For two groups: urban total vs rural

${ }^{d}$ For 3 groups: cities $<100,000$; cities $\geq 100,000$; rural

group $25-34$ to $41.1 \%(28.2 \%$ - $53.9 \%)$ among those 65 years old or older.

People of other nationalities than Polish accounted for only $1.7 \%$ of patients and the imported infections constituted $3.3 \%$ of cases (15/459). Four imported cases concerned persons of a nationality other than Polish (Table 3). Most frequently reported exposures during incubation period were medical procedures (invasive or minor) (Table 3 ) - reported by $62 \%$ of all patients; $60 \%$ of cases were classified as transmitted by medical procedures based on the hierarchy. Medical procedures in hospitals were reported by $37 \%$ of cases and ultimately $35 \%$ of acute hepatitis B cases were attributed to hospital transmission.

In most cases (315/459; 69\%) infected persons reported only one known category of exposure within 16 months prior to the onset of disease, in $49 / 459$ (11\%) indicated two or more exposures of different categories, in $95 / 459$ (21\%) the route of infection was unknown.

Among 170/459 patients hospitalized in the incubation period 11 reported other exposure of higher risk of infection: 5 people among hospitalized injected drugs intravenously, 3 had risky sexual contacts and 3 - tattooing or beauty treatment.

The distribution of presumed transmission routes differed by age among men and women. In those over 40 medical procedures account for over $70 \%$ of infections, while almost no infections are attributed to injecting drug use. In contrast, in the age group 25-39 sexual transmission and injecting drug use play important role assigned to $12.3 \%$ of cases in men in both categories and respectively $16.2 \%$ and $8.1 \%$ in women (Fig. 2). Of note the proportion of cases with unknown transmission route was especially high among men in the younger age group (27\%).

In total, 17 of 459 acute hepatitis B cases were positive for anti-HCV, including two coinfected both with $\mathrm{HCV}$ and HIV. In addition, one person was diagnosed with HBV, HIV and syphilis, with a negative HCV test result. Furthermore, in 3 cases positive for anti-HCV, HCVRNA test results were negative, which indicates $\mathrm{HCV}$ infection in the past. For $32 \%$ of patients with acute hepatitis B, HCV status was unknown. 


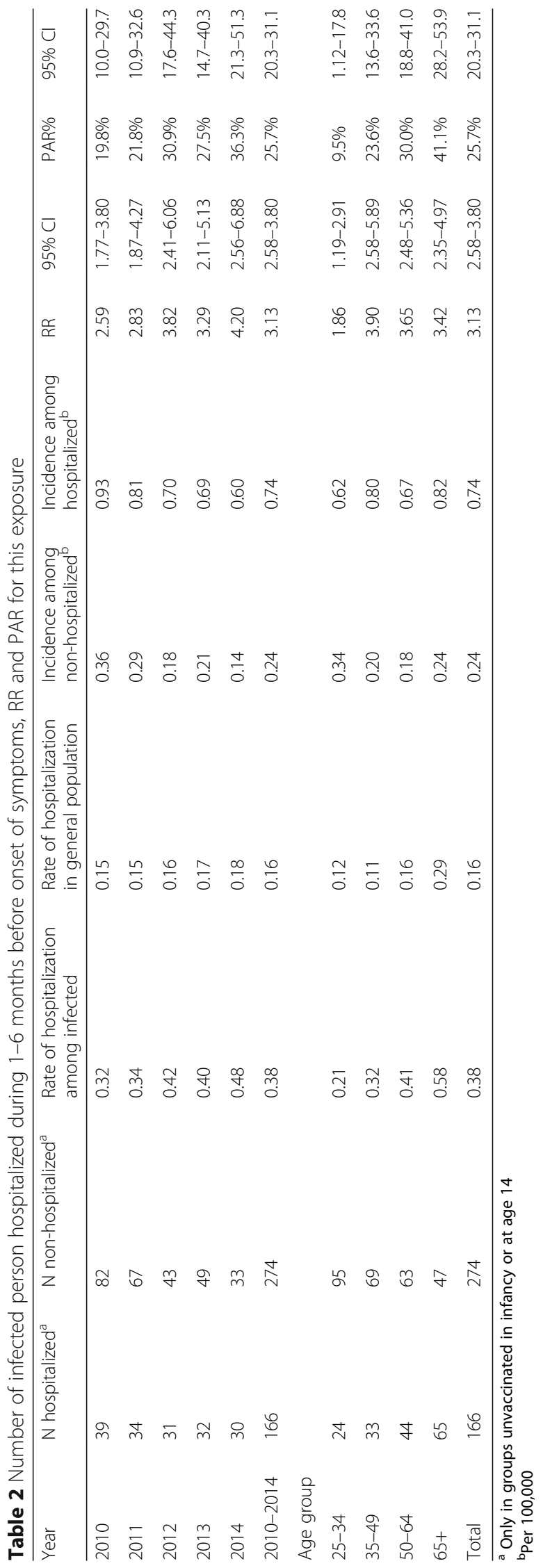


Table 3 Acute hepatitis B in Poland 2010-2014. Distribution of cases by reported exposure and risk factors

\begin{tabular}{|c|c|c|c|}
\hline & & $N$ of cases & $\%$ \\
\hline Total & & 459 & 100.0 \\
\hline Exposure on hepatitis B risk factors & Any medical procedures & 286 & 62.0 \\
\hline \multirow[t]{9}{*}{ (more than one could be mentioned) } & Medical procedures in hospital ${ }^{a}$ & 170 & 37.0 \\
\hline & Invasive procedures & 96 & 20.9 \\
\hline & Medical procedures outside hospital ${ }^{b}$ & 114 & 24.8 \\
\hline & Tattooing/piercing or beauty treatments ${ }^{c}$ & 46 & 10.0 \\
\hline & Injection drug use & 20 & 4.4 \\
\hline & HBV infected sexual partner or risky contacts & 35 & 7.6 \\
\hline & HBV infected household & 25 & 5.4 \\
\hline & Other $^{d}$ & 12 & 2.6 \\
\hline & Unknown/none identified & 97 & 21.1 \\
\hline \multirow[t]{3}{*}{ Migration status } & Polish origin & 448 & 97.6 \\
\hline & immigrant & 8 & 1.7 \\
\hline & unk & 3 & 0.7 \\
\hline \multirow[t]{3}{*}{ Possible importation } & No & 425 & 92.6 \\
\hline & Yes & 15 & 3.3 \\
\hline & unk (transmission unknown) & 19 & 4.1 \\
\hline \multirow[t]{4}{*}{ Vaccination status } & Fully vaccinated & 29 & 6.3 \\
\hline & Incompletely vaccinated & 27 & 5.9 \\
\hline & Not vaccinated & 326 & 71.0 \\
\hline & Vaccination status unknown & 77 & 16.8 \\
\hline \multirow[t]{2}{*}{ Health care worker } & No & 456 & 99.3 \\
\hline & Yes & 3 & 0.7 \\
\hline
\end{tabular}

${ }^{a}$ Includes invasive and minimally invasive surgery, transfusion, dialysis, childbirths, biopsies, endoscopy, injections

${ }^{\mathrm{b}}$ Includes dental treatments and extraction, procedures in outpatient clinics like injections, endoscopy, biopsy and other minimally invasive procedures

'Includes acupuncture, tattoo, piercing, botox cosmetic, mesotherapy, shaving

Includes mother-to child-infections, occupational exposure, stay in prison, sniffing drugs, street fighting and accidental injuries

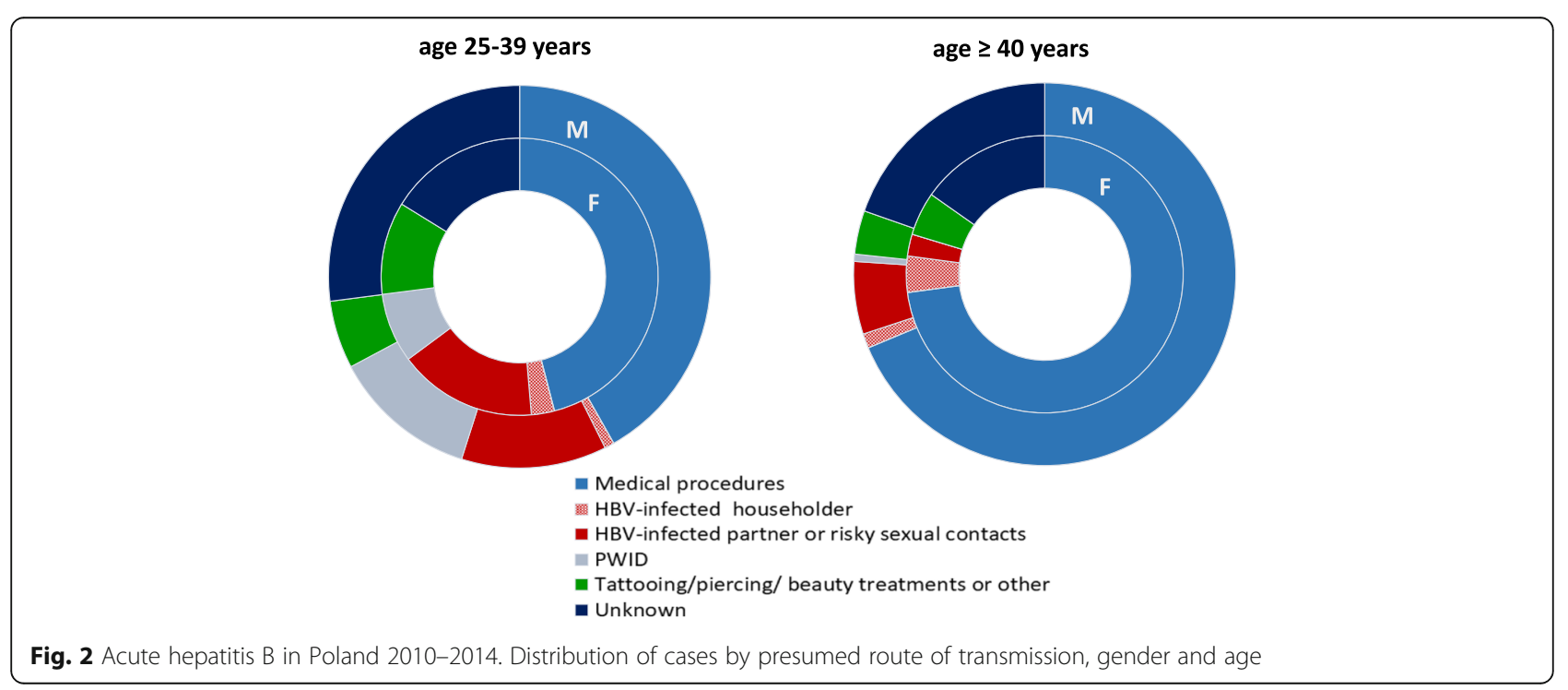


Among 18 people coinfected with $\mathrm{HCV}$ and/or HIV, 6 reported injecting drug use. Further 9 people with HBV/ $\mathrm{HCV}$ coinfection were infected with $\mathrm{HBV}$ by medical procedures and in 3 cases the route of transmission was unknown (all possible exposures were negated).

$\mathrm{HCV}$ co-infections were significantly more common among HBV cases infected by drug use than among those infected another way ( $35 \%$ vs. $3.7 \%, p=0.0001$, in cases with complete information on transmission route and $\mathrm{HCV}$ testing). There were no $\mathrm{HCV}$ co-infections among people infected by sexual contacts, but there were 2 cases of syphilis.

\section{Acute hepatitis B cases in vaccinated birth cohorts}

Of the 19 patients belonging to the vaccinated birth cohorts, 5 were classified as non-responders (fully vaccinated), 3 were vaccinated with 2 doses, 9 were unvaccinated (including 3 immigrants) and in 2 cases documentation was missing. Finally, there was one case of vertical infection that occurred despite prompt administration of HBIG and vaccine after birth.

\section{Discussion}

We report HBV incidence in birth cohorts not covered by the routine vaccination program of new-borns in Poland, by demographic profile. The incidence remains significantly higher among men aged 25-39. Cases among men in this age group are more likely to report injecting drugs and sexual exposures $(12.3 \%$ in each of these categories) as compared to cases amongst older men and amongst women. Further, combining surveillance data with the national health fund data, we identify an increased infection risk associated with hospitalization, suggesting possible transmission in the medical settings.

During 2010-2014, hospitalisation was associated with an over three-fold increase of the risk of acute hepatitis B (RR 3.13; 95\% CI: 2.58-3.80). Based on the population attributable risk we conclude that $25.7 \%$ (95\% CI $20.3-$ 31.1) of cases may be attributed to exposures related to hospitalization, presumably because of poor adherence to universal precautions during medical procedures in hospitals. This is a remarkably high proportion in comparison to the countries in Western Europe and the USA, where the nosocomial transmission is reported in less than $10 \%$ of cases $[3,19]$ and often happen in community settings, nursing homes or dialysis units [4, 20-22]. It calls for revision of the infection control procedures with respect to blood-borne infections in medical settings.

Among cases who were hospitalized during the incubation period $54 \%$ had invasive (infection prone) procedures, i.e. major surgery or dialysis, and in almost half of hospitalized cases the stay in hospital was related to diagnostic procedures (endoscopy, biopsy, CT and MRI with contrast) or the conservative treatment with administration of drugs by injection or infusion. Such structure of exposures among cases may point to transmission during smaller procedures.

Moreover, for $18 \%(84 / 459)$ of acute hepatitis B cases, injections in an outpatient setting or outpatient dental treatment were listed as the only exposure during the period of 1-6 months before the onset of symptoms.

This is in line with other evidence of nosocomial transmission of blood-borne infections, including outbreaks reported from Poland and from other countries [4, 23-26]. The risk of infection associated with small procedures, unsafe injections and poor compliance with safety rules by medical personnel may partly explain the persistence of a large proportion of infections contracted in relation to medical procedures despite the large improvement in the conditions of equipment sterilization and success of the vaccination program. Among small medical procedures, attention should be drawn to the use of medications in multi-dose vials/packages or one vial of medication for more than one patient, inappropriate flushing of i.v. ports and cannulas, use of lancing devices, multiple use of disposable sets for contrast injectors for CT \& MRI, and the insufficient knowledge and experience of personnel in this field [27-29].

Finally, the higher proportion of infections attributed to the health-care associated transmission should be also viewed in the context of background prevalence of chronic (undiagnosed) infection in the general population, following high incidence among young children in 1980s and 1990s.

The highest incidence was recorded among men aged 25-39 years $(0.52$ per 100,000). Moreover, in the age group 25-34 years PAR\% associated with hospitalization was lower than in older age groups - 9.5\% (95\% CI 1.1217.8). It may be associated with a shorter average length of stay in hospital of young people (5 days vs 10 days for persons $>65$ years old and 8 days for all $>24$ years old, but also higher prevalence of other exposures, e.g. injecting drugs or risky sexual contacts.

Within this group (men aged 25-39) the medical procedures represent $42 \%$ of the presumed transmission routes, compared to $60 \%$ in the whole infected population and $57 \%$ of all infected males. Infections among men aged 25-39 acquired by sexual contact (including MSM) and in relation to drug use represent in this group a higher percentage than among males $\geq 40$ ( $25 \%$ vs $7 \%$ ). Additionally, it is possible that the infections related to drug use or sexual transmission, especially among MSM are underreported. Unknown route of infection accounted for a higher proportion of cases among males aged 2539 years than in all cases ( $27 \%$ vs $21 \%)$. 
MSM and PWID are still disproportionately affected by HBV in Europe despite targeted vaccination campaigns in many countries [2,30]. Immunization of these most at risk groups in Poland is not universal. The National Immunization Programme broadly recommends vaccination against hepatitis $B$ for people at increased risk of HBV infection due to lifestyle or individual behaviors and the coverage in this group is likely similar to the general population. Previous research indicated that approximately $50 \%$ of PWID were immunized, but this proportion was below $30 \%$ among birth cohorts not covered by the routine immunization [31]. Targeting these groups would allow to further reduce hepatitis B incidence especially among younger adults. Men born in the 80 's and early 90's should also be targeted for HBV screening due to the possible higher prevalence of chronic HBV infections in this group [6] - acquired perinatally or in early childhood in the period before the introduction of universal vaccinations of newborns. Currently, this is the main gap in testing policy in Poland younger men, especially PWID (current and former) and with risky sexual behaviors, are not targeted effectively by testing practice.

\section{Limitations}

Our study is limited by restricted availability of the population (denominator) data. As we were not able to individually link the surveillance and health fund databases we had to rely on aggregated data. The relative risk for hospitalization may be inaccurate since the recall time for cases was 6 months and the denominator included yearly number of people who were hospitalized. This is likely larger than the source population for our cases, thus we may obtain a conservative estimate of the relative risk.

Moreover, the true risk among exposed (hospitalized) may be higher also due to the fact, that part of the exposed population was actually vaccinated before planned admission and thus not susceptible to the infection. In a study among surgical/gynecological patients, over $60 \%$ were immunized and many of them specifically before the surgery [32]. Again this could bias the association of the recent hospitalization with hepatitis B infection towards the null. We therefore conclude that the risk exists and could only be higher that reported.

Another limitation is that we were not able to calculate the PARs adjusted for other exposures. Unfortunately, data on the population size of PWID and MSM were not available, especially if stratified by recent hospitalization. Finally we relied on the self-reported data. It cannot be excluded that some individuals did not report their sexual or drug injecting exposures. Thus these routes of transmission should be further investigated.

\section{Conclusions}

In conclusion the ongoing HBV incidence may be attributed to two distinct transmission patterns: transmission in medical settings, including undetected outbreaks, and sexual transmission, especially among the men in addition to injecting drug use. Different measures may be appropriate to tackle HBV transmission in these groups, including vaccination campaign for MSM and PWID as well as strengthening the blood-borne infections universal precautions in hospitals. We were able to attribute a quarter of infections to hospitalizations and likely HBV transmission can also occur in the community settings. The ongoing health-care associated transmission indicates the need for continuous staff education and strengthening the monitoring of compliance with current guidelines, especially of injection safety.

\section{Abbreviations \\ $\mathrm{Cl}$ : Confidence interval; CT\&MRI : Computed tomography \& magnetic resonance imaging; HBV: Hepatitis B virus; HCV : Hepatitis C virus; MSM : Men who have sex with men; MTCT : Mother-to-child transmission; NA : Nucleos(t)ide analogues; PAR : Population attributable risk; PWID : People who inject drugs; RR : Risk ratio, relative risk}

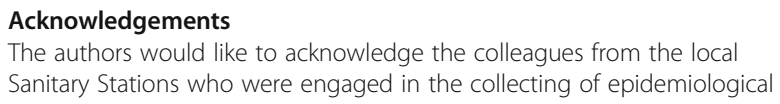

\section{Availability of data and materials}

The dataset supporting the conclusions of this article is available in the Zenodo repository: https://doi.org/10.5281/zenodo.1196322 . Demographic data used in analysis were provided by the Department of Demographic Surveys and Labour Market in Central Statistical Office and were publicly available from: http://demografia.stat.gov.pl/bazademografia/Tables.aspx Data on hospitalization were also publicly available and relevant link was provided in the text

\section{Authors' contributions}

MS - wrote the paper, collected, analysed and interpreted data, KZ collected and analysed data, MR - contributed significantly to the design of the study and to the writing of the manuscript. All authors read and approved the final version of the manuscript.

\section{Ethics approval and consent to participate}

The study is based on surveillance data and as such does not necessitate approval of Ethical Committee. Collection of data on communicable diseases as part of epidemiological surveillance is carried out by the State Sanitary Inspection and $\mathrm{NIPH}-\mathrm{NIH}$ according to the existing legislation: Act of 5th December 2008 on the Prevention and Control of Infections and Infectious Diseases in Humans [33].

Consent for publication

Not applicable.

Competing interests

The authors declare that they have no competing interests. 


\section{Publisher's Note}

Springer Nature remains neutral with regard to jurisdictional claims in published maps and institutional affiliations.

Received: 6 November 2017 Accepted: 26 March 2018

Published online: 10 April 2018

\section{References}

1. World Health Organization (WHO). WHO Hepatitis B fact sheet, Reviewed July 2017. 2017. http://www.who.int/mediacentre/factsheets/fs204/en/. Accessed 2 Aug 2017

2. Duffell EF, Hedrich D, Mardh O, Mozalevskis A. Towards elimination of hepatitis B and C in European Union and European Economic Area countries: monitoring the World Health Organization's global health sector strategy core indicators and scaling up key interventions. Euro Surveill. 2017; 22:pii=30476.

3. European Centre for Disease Prevention and Control (ECDC). Surveillance Atlas of Infectious Diseases. 2017. https://ecdc.europa.eu/en/surveillance-atlasinfectious-diseases. Accessed 2 Mar 2017.

4. Lanini S, Puro V, Lauria FN, Fusco FM, Nisii C, Ippolito G. Patient to patient transmission of hepatitis B virus: a systematic review of reports on outbreaks between 1992 and 2007. BMC Med. 2009;7:15

5. Magdzik W, Czarkowski MP. Epidemiological situation of hepatitis B in Poland in the years 1979-2004. Przegl Epidemiol. 2006;60:471-80.

6. Magdzik W. Hepatitis B epidemiology in Poland, central and Eastern Europe and the newly independent states. Vaccine. 2000:18(Suppl 1):S13-6.

7. Van Damme P, Tormans G, Beutels P, Van Doorslaer E. Hepatitis B prevention in Europe: a preliminary economic evaluation. Vaccine. 1995; 13(SUPPL. 1):S54-7.

8. Magdzik W, Czarkowski MP. Coverage of vaccination against hepatitis B in Poland in 2004. Przegl Epidemiol. 2006;60:185-92.

9. Ganczak M, Ostrowski M, Szych Z, Korzen M. A complete HBV vaccination coverage among polish surgical nurses in the light of anti-HBC prevalence: a cross-sectional sero-prevalence study. Vaccine. 2010;28:3972-6.

10. Ganczak M, Dmytrzyk-Danilow G, Korzen M, Szych Z. A cross-sectional serosurvey on hepatitis B vaccination uptake among adult patients from GP practices in a region of south-West Poland. BMC Public Health. 2015;15: 1060.

11. Stępień M, Piwowarow K, Czarkowski MP. Hepatitis B in Poland in 2014. Przegl Epidemiol. 2016;70:231-41.

12. Hartleb M, Gutkowski K, Zejda JE, Chudek J, Więcek A. Serological prevalence of hepatitis $B$ virus and hepatitis $C$ virus infection in the elderly population: polish nationwide survey - PolSenior. Eur J Gastroenterol Hepatol. 2012;24:1288-95.

13. Pszenny A, Hreńczuk M, Czerwiński J, Danielewicz R, Małkowski P. HBV and HCV infections among deceased organ donors in Poland. Probl Hig Epidemiol. 2012;93:579-85.

14. Grabarczyk P, Kopacz A, Sulkowska E, Kubicka-Russel D, Mikulska M, Brojer E, et al. Blood donors screening for blood born viruses in Poland. Przegl Epidemiol. 2015:69:473-5.

15. Commission of the European Communities. Commision Decision of 28/IV/ 2008 amending Decision 2002/253/EC laying down case definitions for reporting communicable diseases to the Community network under Decision No 2119/98/EC of the European Parliament and of the Council. 2008. http://eur-lex.europa.eu/legal-content/EN/TXT/?qid= 1469622720178\&uri=CELEX:32008D0426. Accessed 27 Jul 2017.

16. Goldstein ST, Alter MJ, Williams IT, Moyer LA, Judson FN, Mottram K, et al. Incidence and risk factors for acute hepatitis B in the United States, 19821998: implications for vaccination programs. J Infect Dis. 2002;185:713-9.

17. Allain JP, Candotti D. Hepatitis B virus in transfusion medicine: still a problem? Biologicals. 2012;40:180-6

18. Rockhill B, Newman B, Weinberg C. Use and misuse of population attributable fractions. Am J Public Health. 1998:88:15-9.

19. Centers for Disease Control and Prevention's (CDC). Surveillance for Viral Hepatitis - United States, 2013. https://www.cdc.gov/hepatitis/statistics/ 2013surveillance/commentary.htm\#hepatitisB. Accessed 2 Aug 2017.

20. Duffell EF, Milne LM, Seng C, Young Y, Xavier S, King S, et al. Five hepatitis B outbreaks in care homes in the UK associated with deficiencies in infection control practice in blood glucose monitoring. Epidemiol Infect. 2011;139: 327-35.
21. Fabrizi F, Dixit V. Messa P, Martin P. Transmission of hepatitis B virus in dialysis units: a systematic review of reports on outbreaks. Int J Artif Organs. 2015;38:1-7

22. Pourkarim MR, Verbeeck J, Rahman M, Amini-Bavil-Olyaee S, Forier AM, Lemey $P$, et al. Phylogenetic analysis of hepatitis B virus full-length genomes reveals evidence for a large nosocomial outbreak in Belgium. J Clin Virol. 2009:46:61-8.

23. Centers for Disease Control and Prevention (CDC). Investigation of viral hepatitis infections possibly associated with health-care delivery-New York City, 2008-2011. MMWR Morb Mortal Wkly Rep 2012;61:333-338.

24. Kolosova A, Gasparovic J. Viral hepatitis B and C outbreak related to parenteral treatment at an oncological department in Slovakia. J Hosp Infect. 2016:93:211-4.

25. Stepien M, Rosinska M. Hepatitis C outbreaks in Poland in 2003-2013. Medical procedures as a dominant route of HCV transmission. Przegl Epidemiol. 2015;69:465-90.

26. Czerwinski M, Grabarczyk P, Stepien M, Kubicka-Russel D, Tkaczuk K, Brojer E, et al. What weighs more-low compliance with self-deferral or minor medical procedures? Explaining the high rate of hepatitis $C$ virus windowperiod donations in Poland: HCV window-period donations in Poland. Transfusion (Paris). 2017;57:1998-2006

27. Centers for Disease Control and Prevention (CDC). Healthcare-Associated Hepatitis B and C Outbreaks Reported to the Centers for Disease Control and Prevention (CDC) 2008-2015. 2016. www.cdc.gov/hepatitis/outbreaks/ pdfs/healthcareinvestigationtable.pdf.

28. Lanini S, Garbuglia AR, Puro V, Solmone M, Martini L, Arcese W, et al. Hospital cluster of HBV infection: molecular evidence of patient-to-patient transmission through lancing device. PLoS One. 2012;7:e33122.

29. Westbrook Jl, Rob MI, Woods A, Parry D. Errors in the administration of intravenous medications in hospital and the role of correct procedures and nurse experience. BMJ Qual Saf. 2011;20:1027-34.

30. Baars JE, Boon BJ, Garretsen HF, van de Mheen D. The reach of a hepatitis B vaccination programme among men who have sex with men. Eur J Pub Health. 2011;21:333-7

31. Rosinska M. [Estimated HCV, HBV and HIV prevalence in injecting drug users population in Gdansk and in Krakow, 2009.]. 2009. http://www.cinn.gov.pl/ portal?id=166349. Accessed 20 Feb 2017.

32. Ganczak M, Szych Z, Korzen M. Preoperative vaccination for HBV at polish hospitals as a possible public health tool to limit the spread of the epidemic: a cross-sectional study. Vaccine. 2009;27:3969-74

33. [Act of 5th December 2008 on the Prevention and Control of Infections and Infectious Diseases in Humans]. Available from: http://ppiop.rcl.gov.pl/index. php? $r=$ skorowidz/view\&id $=5455$

\section{Submit your next manuscript to BioMed Central and we will help you at every step:}

- We accept pre-submission inquiries

- Our selector tool helps you to find the most relevant journal

- We provide round the clock customer support

- Convenient online submission

- Thorough peer review

- Inclusion in PubMed and all major indexing services

- Maximum visibility for your research

Submit your manuscript at www.biomedcentral.com/submit 